\title{
Pyocyanin Restricts Social Cheating in Pseudomonas aeruginosa
}

\author{
Paulina Castañeda-Tamez', Jimena Ramírez-Peris 1,2 , Judith Pérez-Velázquez ${ }^{3,4}$, \\ Christina Kuttler ${ }^{4}$, Ammar Jalalimanesh ${ }^{4,5}$, Miguel Á. Saucedo-Mora', \\ J. Guillermo Jiménez-Cortés ${ }^{1}$, Toshinari Maeda ${ }^{6}, Y$ Yael González $^{1}$, María Tomás $^{7}$, \\ Thomas K. Wood ${ }^{8}$ and Rodolfo García-Contreras ${ }^{1 *}$
}

'Department of Microbiology and Parasitology, Faculty of Medicine, Universidad Nacional Autónoma de México, Mexico City, Mexico, ${ }^{2}$ Instituto Nacional de Psiquiatría Ramón de la Fuente Muñiz, Mexico City, Mexico, ${ }^{3}$ Institute of Computational Biology, Helmholtz Zentrum München, Deutsches Forschungszentrum für Gesundheit und Umwelt (GmbH), Neuherberg, Germany, ${ }^{4}$ Zentrum Mathematik, Technische Universität München, Munich, Germany, ${ }^{5}$ Iranian Research Institute for Information Science and Technology (IRANDOC), Tehran, Iran, ${ }^{6}$ Department of Biological Functions Engineering, Kyushu Institute of Technology, Kitakyushu, Japan, ${ }^{7}$ Department of Microbiology, Instituto de Investigación Biomédica de A Coruña, Complexo Hospitalario Universitario de A Coruña, SERGAS, Universidade da Coruña, A Coruña, Spain, ${ }^{8}$ Department of Chemical Engineering, The Pennsylvania State University, State College, PA, United States

OPEN ACCESS

Edited by:

Satoshi Tsuneda,

Waseda University, Japan

Reviewed by:

Nina Jagmann,

Universität Münster, Germany

David Daudé,

Gene \& GreenTK, France

${ }^{*}$ Correspondence: Rodolfo García-Contreras

rgarc@bq.unam.mx

Specialty section:

This article was submitted to Microbial Physiology and Metabolism,

a section of the journal

Frontiers in Microbiology

Received: 09 February 2018

Accepted: 04 June 2018

Published: 27 June 2018

Citation:

Castañeda-Tamez P, Ramírez-Peris J,

Pérez-Velázquez J, Kuttler $C$, Jalalimanesh A, Saucedo-Mora MÁ,

Jiménez-Cortés JG, Maeda T,

González Y, Tomás M, Wood TK and

García-Contreras R (2018) Pyocyanin

Restricts Social Cheating

in Pseudomonas aeruginosa.

Front. Microbiol. 9:1348.

doi: 10.3389/fmicb.2018.01348
Quorum sensing (QS) in Pseudomonas aeruginosa coordinates the expression of virulence factors, such as exoproteases and siderophores, that are public goods utilized by the whole population of bacteria, regardless of whether they invested or not in their production. These public goods can be used by QS defective mutants for growth, and since these mutants do not contribute to public goods production, they are considered social cheaters. Pyocyanin is a phenazine that is a toxic, QS-controlled metabolite produced by $P$. aeruginosa. It is a redox-active compound and promotes the generation of reactive oxygen species; it also possesses antibacterial properties and increases fitness in competition with other bacterial species. Since QS-deficient individuals are less able to tolerate oxidative stress, we hypothesized that the pyocyanin produced by the wild-type population could promote selection of functional QS systems in this bacterium. Here, we demonstrate, using competition experiments and mathematical models, that, indeed, pyocyanin increases the fitness of the cooperative QS-proficient individuals and restricts the appearance of social cheaters. In addition, we also show that pyocyanin is able to select QS in other bacteria such as Acinetobacter baumannii.

Keywords: public goods, pyocyanin, social cheating, quorum sensing (QS), oxidative stress, policing

\section{INTRODUCTION}

Pseudomonas aeruginosa uses QS to estimate its population density and to reprogram its gene expression and behavior accordingly. This system relies on the continuous production of small chemical signals known as autoinducers which are produced at a low rate until they accumulate and interact with their receptors. The receptors are activated by signal binding and induce transcription of the promoters of the genes encoding the enzymes that synthesize the autoinducers, which results in a sudden increase in autoinducer concentrations and receptor activation. Receptor activation also allows for increased transcription of several other genes allowing the coordination 
of the expression of cooperative behaviors like the production of costly exoproducts such as exoenzymes and siderophores (Popat et al., 2015; García-Contreras, 2016). In P. aeruginosa, at least four interconnected QS systems exist; for two of them, LasIR and RhlIR, the autoinducer signals are $N$-acyl homoserine lactones while for the third system, PQS, hydrophobic quinolones are used, and for the fourth system, IQS, which is active mainly under conditions of phosphate starvation, 2-(2-hydroxyphenyl)thiazole-4-carbaldehyde is used as the signal (Lee and Zhang, 2015). These subsystems are hierarchically organized, with LasIR at the top, in charge of the activation of the other systems (Castillo-Juarez et al., 2015; Papenfort and Bassler, 2016). In general, the costly exoproduct production which QS regulates can benefit all the individuals in the population, regardless if they invested in their production or not; hence, these exoproducts are public goods. Since the production of public goods is costly, the individuals that use them without contributing to their production are social cheaters (Diggle et al., 2007) and can invade the population, causing a tragedy of the commons (Sandoz et al., 2007). It has been demonstrated in P. aeruginosa that cheating behavior exists for both exoprotease production (Diggle et al., 2007) and for the production of the main siderophore pyoverdine (Kummerli et al., 2009). In the first case, cheaters (mutants deficient in las $R$ ) naturally emerge in cultures with protein as the sole carbon source and also have been isolated from several environments such as the lungs of cystic fibrosis patients (Sandoz et al., 2007; Hoffman et al., 2009; Dandekar et al., 2012; GarcíaContreras et al., 2015a). In the second case, mutants unable to produce pyoverdine are selected in iron deficient media (Dumas and Kummerli, 2012).

However, although cheating exists in nature, QS systems that regulate public goods are common; hence, factors that counteract the effects of social cheaters should exist. For P. aeruginosa, those mechanisms include the growth of bacteria in conditions that allow the physical separation of cooperators and cheaters, decreasing the cheater's fitness (Hense et al., 2007; Mund et al., 2016). This occurs during the growth in highly viscous medium, since viscosity limits diffusion of public goods such as siderophores (Kummerli et al., 2009). Another way to counteract social cheating is to combine the assimilation of protein, which depends on the production of exoproteases that are exploited by cheaters, with the assimilation of adenosine, whose catabolism is also QS-dependent, but in contrast to the extracellular protein, it is mediated by intracellular private enzymes (Dandekar et al., 2013). Finally environmental factors such as the presence of toxic compounds such as $\mathrm{H}_{2} \mathrm{O}_{2}$ (García-Contreras et al., 2015b) and HCN (Wang et al., 2015), and the presence of some temperate phages (Saucedo-Mora et al., 2017) also restrict social cheating, since QS deficient mutants are more sensitive to stress due a lower expression of anti-oxidant enzymes such as catalase and superoxide dismutase (Hassett et al., 1999), and likely due to a lack of modifications that enhance membrane tolerance to stress (Davenport et al., 2015). In addition, a lower expression of cyanide insensitive cytochrome oxidase may contribute to their higher sensitivity toward HCN (Wang et al., 2015).

One of the main QS-controlled virulence factors are the phenazines, dibenzo annulated pyrazines with diverse activities such as: (i) cell signaling (Dietrich et al., 2006), (ii) biofilm formation (Ramos et al., 2010; Wang et al., 2011), (iii) survival in anaerobiosis (Wang et al., 2010), (iv) fitness in the presence of competitor bacteria (Smalley et al., 2015), (v) resistance to gallium nitrate (García-Contreras et al., 2013; Rezzoagli et al., 2017) and other toxic metals such as silver (Muller and Merrett, 2014), and (vi), damage to host cells (Rada and Leto, 2013), among others.

Pyocyanin, one of the main phenazines of P. aeruginosa, is produced by several clinical strains from pulmonary and extrapulmonary infections (Schaber et al., 2004; García-Contreras et al., 2015b; Guendouze et al., 2017) as well as in environmental strains (Grosso-Becerra et al., 2014) and is found in high concentrations [up to $100 \mu \mathrm{M}$ (Caldwell et al., 2009) in the lung of cystic fibrosis patients]. Due to its redox activity, pyocyanin increases the production of reactive oxygen species by donating electrons to oxygen, thereby producing hydrogen peroxide. Moreover, pyocyanin also depletes the pools of antioxidant molecules like glutathione. Taking these results into account as well as the fact that QS-defective mutants are less able to cope with oxidative stress (García-Contreras et al., 2015b) and likely have membranes more sensitive to oxidative stress than QS-proficient individuals due lower levels of cyclopropanation and lower levels of fatty acid saturation (Davenport et al., 2015), we hypothesized that pyocyanin may differentially affect this population and may act as a policing metabolite. To test this hypothesis, we performed competition experiments and developed mathematical models; our results show that indeed the production of this metabolite selects for the presence of QS.

\section{MATERIALS AND METHODS}

\section{Bacterial Strains and Growth Conditions}

Pseudomonas aeruginosa PA14, and the lasR rhlR mutant were donated by Dr. You-Hee Cho from the College of Pharmacy, CHA University, South Korea (Park et al., 2005). The PA14 phzM mutant (40343 from the collection) was provided by Dr. Frederick Ausubel from the Harvard Medical School (Liberati et al., 2006). In order to avoid differences between PA14 genetic backgrounds, the phzM mutation was incorporated into the PA14 strain from South Korea by phage transfection using the DMS3 phage (Budzik et al., 2004). The Acinetobacter baumannii mutant with the abaI gene deleted was generated using the pMo130 plasmid (Hamad et al., 2009) which carries the aphA gene that provides kanamycin resistance, the $x y l E$ gene that allows visual detection of the mutants, and the modified $s a c B$ gene that allows the resolution of the co-integrants. The mutants were confirmed by sequencing and real-time PCR with Taqman probes (see Supplementary Table S1 for the primer sequences).

Pre-cultures of all strains were grown in $\mathrm{LB}$ at $37^{\circ} \mathrm{C}$ with $200 \mathrm{rpm}$ shaking for $\sim 16 \mathrm{~h}$. For the $p h z M$ mutant, gentamicin at $15 \mu \mathrm{g} \mathrm{mL}^{-1}$ was added. The pre-cultures were then used for the inoculation of flasks with M9 minimal medium supplemented with $0.25 \%$ of sodium caseinate as the sole carbon source (M9 caseinate medium) with or without exogenous protease 
(type XIV p5147, from SIGMA at 1 unit/ml) or with the same medium with $0.25 \%$ of casamino acids as a sole carbon source or with LB medium, and the cultures were grown under the same conditions. Growth was monitored by recording the increase of the turbidity $(600 \mathrm{~nm})$ with a spectrophotometer (UV-1800, Shimadzu).

\section{Bactericidal Effect of Pyocyanin}

To evaluate the bactericidal effect of pyocyanin, wild-type, the lasR rhlR mutant and the phzM mutant cells were cultured in LB to turbidity at $600 \mathrm{~nm}$ of 1.0 , then samples $(1 \mathrm{~mL})$ were taken and pyocyanin at 50 or $100 \mu \mathrm{M}$ was added. Cells were exposed for $30 \mathrm{~min}$, and viable counts were used to determine the degree of survival.

\section{Effect of Pyocyanin in the Selection of Social Cheaters}

Single colonies were inoculated into LB medium to form precultures that were transferred to $\mathrm{M} 9$ caseinate medium (initial turbidity of $\sim 0.05$ at $600 \mathrm{~nm}$ ); each culture was incubated for $24 \mathrm{~h}$ and was used for inoculating subsequent cultures (also at an initial turbidity of $\sim 0.05$ ) with or without the addition of $100 \mu \mathrm{M}$ pyocyanin (added to the medium before inoculation). The percentage of protease-less producer colonies after each $24 \mathrm{~h}$ culture was evaluated by plating colonies (between 25 and 50 per culture) and visualizing the casein degradation halo in LB plates with $3 \%$ skimmed milk.

\section{Competition Experiments}

Cultures in M9 caseinate medium were inoculated with different proportions of the wild-type PA14 strain and the lasR rhlR mutant or the phzM mutant and the lasR rhlR mutant. For all cases, enough bacteria to achieve an initial turbidity at $600 \mathrm{~nm}$ of $\sim 0.05$ was used, then the cultures were grown under the same conditions detailed above. Samples of each culture were taken after $0,4,6,10$, and $24 \mathrm{~h}$ of cultivation and were used to isolate colonies. The proportion of PA14 and the las $R$ rhlR mutant was determined by evaluating casein degradation.

For evaluating the effect of pyocyanin in the competition between the lasR $r h l R$ mutant and the phzM mutant, pyocyanin was purified by the extraction with chloroform and $0.2 \mathrm{M} \mathrm{HCl}$ (Essar et al., 1990), and its purity was verified by comparing its absorbance spectrum with commercial pyocyanin (SIGMA, St. Louis, MO, United States). Pyocyanin (neutralized with $\mathrm{NaOH}$ ) was added at $25 \mu \mathrm{M}$ to the cultures, and at $4,6,10$, and $24 \mathrm{~h}$, samples were taken to estimate the proportion of QS mutant and phzM populations.

\section{A. baumannii Competition}

Acinetobacter baumannii ATCC-17978 and its isogenic abaI mutant were cultured in LB medium until they reached to the early stationary phase (turbidity at $600 \mathrm{~nm} \sim 2.0$ ), and then $500 \mu \mathrm{l}$ of each culture was mixed in $2 \mathrm{~mL}$ tubes with or without $50 \mu \mathrm{M}$ of pyocyanin and incubated for $20 \mathrm{~min}$ under the same conditions. Samples of the mixtures were taken before adding pyocyanin and after the incubations for isolating colonies that were then plated on blood agar plates; plates were incubated for $16 \mathrm{~h}$ at $37^{\circ} \mathrm{C}$ and then the identity of these colonies was determined by PCR. A total of 140 colonies were screened using two PCR reactions: one for the identification of the $a b a I$ gene and the other for the house keeping gene $p b p C$ using primers $5^{\prime}$-CCAATATCATTGGTTGTGCC and 5' TCGTAATGAGTTGTTTTGCG that yield a product of $255 \mathrm{bp}$ for $a b a I$, and the primers $5^{\prime}$-TTTGACTGGGATGGTATTCG and $5^{\prime}$-GGAATCTGGGTGCTATTCAT that yield a product of $419 \mathrm{bp}$ for $p b p C$.

\section{Pyocyanin and Exoprotease Assay}

Pyocyanin concentrations were determined spectrophotometrically after the extraction with chloroform and $0.2 \mathrm{M} \mathrm{HCl}$ (Essar et al., 1990). The absorbance at $520 \mathrm{~nm}$ was used with an $\varepsilon$ of $2.46 \mathrm{mM}^{-1} \mathrm{~cm}^{-1}$ (O'Malley et al., 2004). Elastase was determined by quantifying the production of Congo red from the hydrolysis elastine-Congo red complex (SIGMA) (Ohman et al., 1980), and alkaline protease concentrations were determined by the production of remazol brilliant blue $\mathrm{R}$ from the hydrolysis of the Hide-Remazol Brilliant Blue $\mathrm{R}$ powder (SIGMA) (Howe and Iglewski, 1984).

\section{Catalase Assay}

Catalase activity was determined by recording the conversion of $\mathrm{H}_{2} \mathrm{O}_{2}$ to $\mathrm{H}_{2} \mathrm{O}+\mathrm{O}_{2}$, spectrophotometrically at $240 \mathrm{~nm}$ and $37^{\circ} \mathrm{C}$ in Tris (50 mM at $\mathrm{pH} 7$ ). PA14 and the QS mutants cells were grown in M9 medium with $0.25 \%$ of casamino acids to the middle exponential phase (turbidity $\sim 1.0$ at $600 \mathrm{~nm}$ ), then $1 \mathrm{~mL}$ of each culture was harvested by centrifugation; the cell pellets were washed in the same reaction buffer once, resuspended in $250 \mu \mathrm{L}$ of the buffer, and half of the cells were mixed with $125 \mu \mathrm{L}$ of a permeabilization solution $(100 \mathrm{mM}$ dibasic sodium phosphate, $20 \mathrm{mM} \mathrm{KCl}, 2 \mathrm{mM} \mathrm{MgSO} 4,0.8 \mathrm{mg} / \mathrm{mL}$ CTAB, $0.4 \mathrm{mg} / \mathrm{mL}$ sodium deoxycholate), incubated for $20 \mathrm{~min}$ at $37^{\circ} \mathrm{C}$, and then used to determine activity. In parallel, $1 \mathrm{~mL}$ of each culture was exposed to $25 \mu \mathrm{M}$ of pyocyanin at $37^{\circ} \mathrm{C}$ for $30 \mathrm{~min}$ and after that, cell pellets were obtained and the procedures described before were followed to determine the effect of pyocyanin in the catalase activity. Protein concentrations were determined by the Bradford method, and catalase activity was expressed as nanomol of $\mathrm{H}_{2} \mathrm{O}_{2}$ degraded per minute per mg of protein.

\section{Statistical Analysis}

All experiments were done at least in triplicate. Statistical significances for Figures 3, 4, 6 were evaluated by using a Student's two-tailed test and considered significant if $P<0.05$. Statistical significance for Figure 5 was evaluated by a chisquared test and considered significant if $P<0.05$.

\section{Mathematical Modeling}

A spatial stochastic mathematical model was developed to further explore the role of pyocyanin in the selection of the QS phenotype during the competition experiments. A deterministic mathematical model was used to compute some of the parameters involved. A detailed description of the models can be found in the Supplementary Materials. 


\section{RESULTS}

\section{Pyocyanin Selects the Wild-Type Phenotype in Competition With Non-protease Producer Mutants}

Our first approximation to evaluate factors that stabilize QS in $P$. aeruginosa was to test their effect in the outcome of competition experiments between the PA14 wild-type strain and non-protease producer, the las $R$ rhlR mutant, in medium with caseinate as the sole carbon source. In this medium, the only way mutants can grow is by utilizing the peptides and amino acids produced by the hydrolysis of casein by the QScontrolled exoproteases synthetized and exported by the wildtype strain; hence, lasR rhlR mutants behaved as social cheaters that exploit the cooperator (wild-type) individuals (Diggle et al., 2007; García-Contreras et al., 2015b). Growth curves of PA14, the las $R$ rhlR mutant, and their mixtures containing an initial proportion of 10,50 , and $90 \%$ of the las $R$ rhlR mutant population confirmed that the lasR rhlR mutant was unable to grow in caseinate as the sole carbon source after $24 \mathrm{~h}$ of cultivation and show that increasing the initial proportion of the lasR rhlR mutant decreased the growth yields of the cultures after $24 \mathrm{~h}$ of cultivation (Supplementary Figure S1). In addition, the overall growth rate of the population also decreased with increasing initial amounts of the las $R$ rhlR mutant, indicating that the presence of QS cheaters is a burden for the system's growth.

In agreement with previous findings, the proportion of the lasR rhlR mutant populations substantially increased with time (Mellbye and Schuster, 2011; Gerdt and Blackwell, 2014). After $24 \mathrm{~h}$ of culture, the percentage of the lasR rhlR mutant was of approximately 50, 75, and $97 \%$ when the initial proportions were approximately 10, 50, and 90\%, respectively (Figure 1), validating that the las $r h l R$ mutant grows at the expense of the wild-type that produced exoprotease under this condition.

These equilibrium final proportions were reached around $10 \mathrm{~h}$ and remained mostly stable (the mutant fraction only increased slightly) after $24 \mathrm{~h}$. Critically, the stable final proportions consisted of lower amounts of the mutant when the amount of mutants in the inoculum was lower; hence, the final proportions depended on the initial proportions, indicating that probably factors produced by the cooperative PA14 individuals were restricting further increases in the mutant proportion. In our experiments, it was clear that the production of a potential toxic metabolite by the wild-type PA14 population, the phenazine pyocyanin, was high and its amount positively correlated with a higher initial wild-type proportion as expected (Figure 2). The production of pyocyanin is positively regulated by the LasR/RhlR systems (Dekimpe and Deziel, 2009), and the mutant we used was unable to produce pyocyanin (García-Contreras et al., 2015b).

Therefore, competition experiments between a nonpyocyanin producer but QS-active strain, the phzM mutant, and the lasR rhlR mutant were conducted; the results indicated that after 6 and $10 \mathrm{~h}$, the relative frequency of the las $\mathrm{rhl} R$

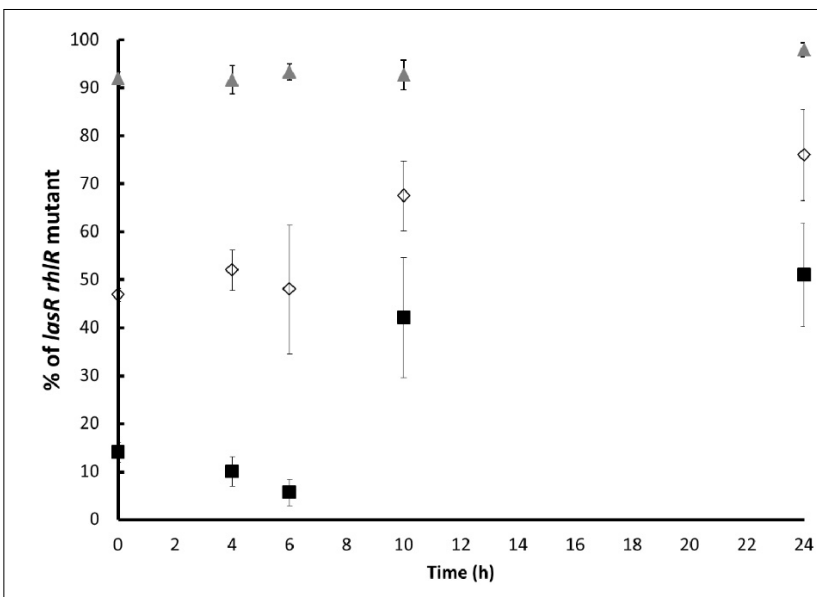

FIGURE 1 | Proportion of the lasR rhIR mutant during competition with the PA14 wild-type in caseinate medium. The initial proportion of the mutant was approximately 10\% (black squares), 50\% (white diamonds), and 90\% (gray triangles). Experiments were performed in quintuplicate, and the average \pm SEM are shown. The differences between all the proportions at the initial time vs. all the proportions at $10 \mathrm{~h}$ are significant, except for the proportion of $90 \%$. The differences between all the proportions at the initial time vs. all the proportions at $24 \mathrm{~h}$ were significant, and no significant difference was found between 10 and $24 \mathrm{~h}$ for any case $(P<0.05$ in a one tailed $T$-student test).

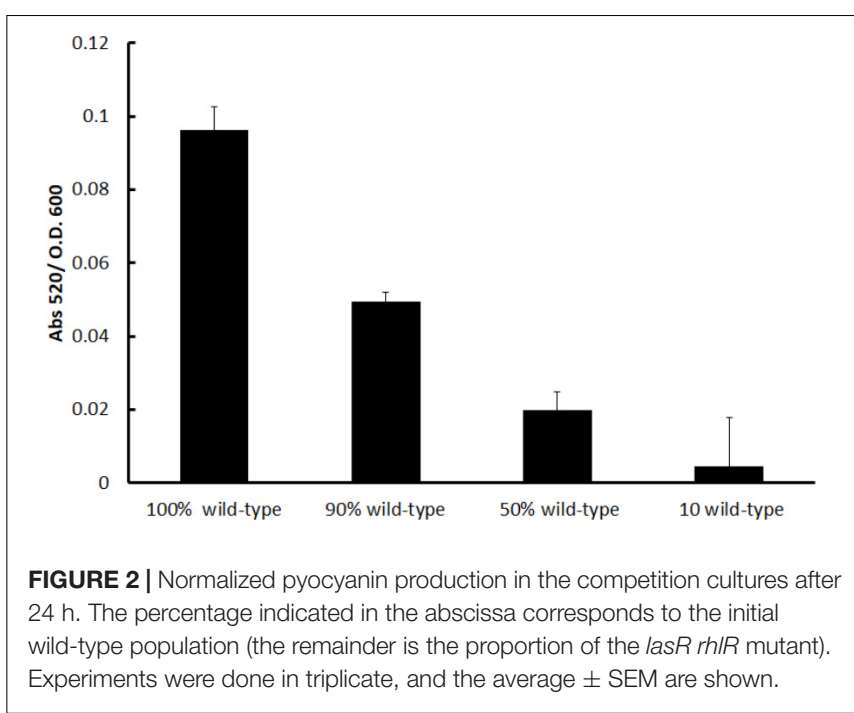

mutant was significantly higher than when they were competing against PA14 when the initial mutant proportion was $10 \%$ (Figure 3A). In agreement, the addition of pyocyanin to the phzM vs. lasR rhlR mutant competition experiments strongly decreased the frequency of the lasR $r h l R$ mutant at $10 \mathrm{~h}$ for initial proportions of $10 \%$ (Figure 3B) and $90 \%$ of mutants (Figure 3C), demonstrating that in addition to $\mathrm{HCN}$, pyocyanin was also capable of restricting cheating (lasR rhlR mutant).

Control growth experiments of the three strains (PA14 wildtype, the lasR rhlR mutant, and the phzM mutant) in M9 medium with $0.25 \%$ of casamino acids as the sole carbon source and with $0.25 \%$ caseinate plus exogenous protease showed almost 

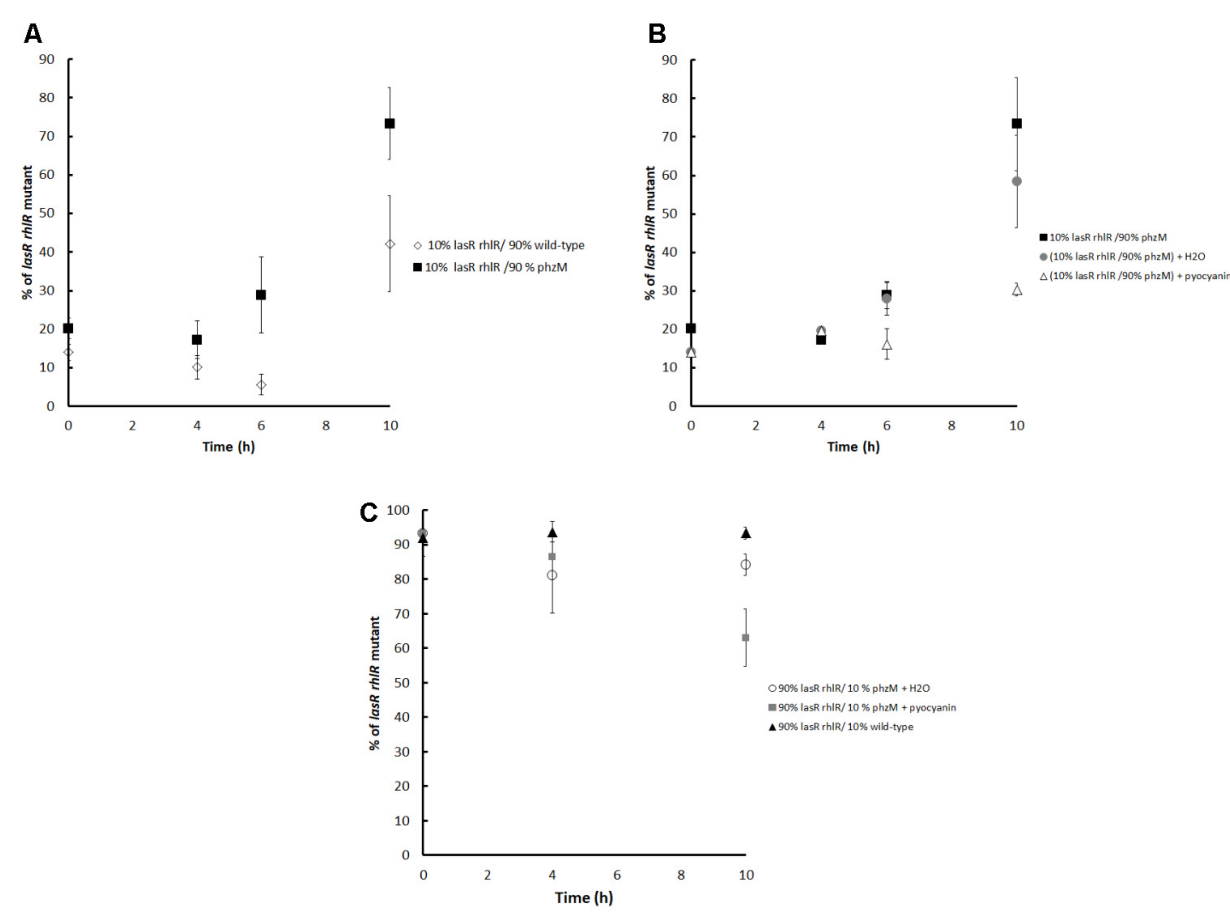

FIGURE 3 | (A) Competitions between the lasR rhIR mutant (cheater) and the phzM mutant (unable to make pyocyanin) grown in M9 caseinate medium. For comparison, competition between the PA14 wild-type and the las $R$ rhlR mutant is also shown. The initial proportion of the las $R$ rhlR mutant was $\sim 10 \%$. (B) Effect of adding pyocyanin $\left(50 \mu \mathrm{M}\right.$ at $4 \mathrm{~h}$ ) to the competition experiments between the las $R$ rhlR mutant and the phzM mutant. The negative controls are $\mathrm{H}_{2} \mathrm{O}$ or no compound added, and the initial percentage of the las $R$ rhlR mutant was $\sim 10 \%$. (C) Competitions between the PA14 wild-type and the las $R$ rhIR mutant and between the phzM and las $R$ rhIR mutants with and without pyocyanin when the initial proportion of the las $R$ rhlR mutant was $\sim 90 \%$. Average of 4 independent cultures \pm SEM are shown. For (A), differences between the proportions of both curves at 6 and $10 \mathrm{~h}$ are statistically significant $(P<0.05$ in a two tailed $T$-student test). For (B), differences between the control curve and the curve with added $\mathrm{H}_{2} \mathrm{O}$ are not significant, but they are statistically significant when compared to the curve with added pyocyanin at 6 and $10 \mathrm{~h}$. For (C), the differences between the three curves are significant at $10 \mathrm{~h}(P<0.05$ in a two tailed $T$-student test).

TABLE $1 \mid P$. aeruginosa strains characteristics.

\begin{tabular}{|c|c|c|c|c|c|c|c|}
\hline Strain & $\begin{array}{l}\text { Growth rate } \\
\text { in caseinate } \\
0.25 \%\left(\mathrm{~h}^{-1}\right)\end{array}$ & $\begin{array}{l}\text { Growth rate in } \\
\text { caseinate } 0.25 \% \\
\text { +protease }\left(\mathrm{h}^{-1}\right)\end{array}$ & $\begin{array}{l}\text { Growth rate in } \\
\text { casamino acids } \\
0.25 \%\left(\mathrm{~h}^{-1}\right)\end{array}$ & $\begin{array}{l}\text { Pyocyanin } \\
(\mu \mathrm{M})\end{array}$ & $\begin{array}{l}\text { Exoprotease } \\
\text { skimmed milk }\end{array}$ & $\begin{array}{l}\text { Elastase } \\
\text { (Abs } 595 \mathrm{~nm} \text { ) }\end{array}$ & $\begin{array}{l}\text { Collagenase } \\
\text { (Abs } 495 \mathrm{~nm} \text { ) }\end{array}$ \\
\hline PA14 wt & $0.39 \pm 0.12$ & $0.73 \pm 0.05$ & $1.06 \pm 0.02$ & $127 \pm 0.8$ & ++ & $3.36 \pm 0.49$ & $2.4 \pm 0.42$ \\
\hline phzM & $0.12 \pm 0.02$ & $0.73 \pm 0.04$ & $0.99 \pm 0.04$ & $1 \pm 0.3$ & + & $1.18 \pm 0.24$ & $1.06 \pm 0.9$ \\
\hline lasR rhlR & Do not growth & $0.79 \pm 0.03$ & $1.07 \pm 0.026$ & Not determined & - & Not determined & Not determined \\
\hline
\end{tabular}

no difference in the growth rates of the strains Table 1 and Supplementary Figures S2A,B.

\section{Pyocyanin Toxicity}

In agreement with the competition experiments, the bactericidal effect of pyocyanin on the las $R$ rhlR mutant was greater than its effect on PA14 and the phzM mutant (Figure 4A). Also the addition of pyocyanin was able to significantly induce the activity of catalase in PA14 cultures but not in the lasR rhlR mutant (Figure 4B), indicating that phenazine was indeed inducing production of $\mathrm{H}_{2} \mathrm{O}_{2}$, which then induced catalase (essentially a private good) in the QS-proficient cells but not in the lasR rhlR mutant. This increase in catalase activity likely allowed PA14 to detoxify $\mathrm{H}_{2} \mathrm{O}_{2}$ faster than the lasR rhlR mutant.

\section{Mathematical Model of the Competitions}

In order to further explore the above-described phenomena, two mathematical models were created: one based on ordinary differential equations (ODEs) and an agent-based model (ABM). The use of ODEs allowed us to verify that the most essential mechanisms of the system had been taken into account as the model could be fitted well to the observed data (Supplementary Materials and Supplementary Figure SM1). Furthermore, the fitting procedure provided quantitative information about several of the parameters involved, such as production rates. Therefore, the same mechanisms were taken into account to set up an ABM.

An $\mathrm{ABM}$ is a mathematical spatial model which allows one to simulate the spatio-temporal dynamics of agents (in this case bacterial cells) under diverse conditions. It 

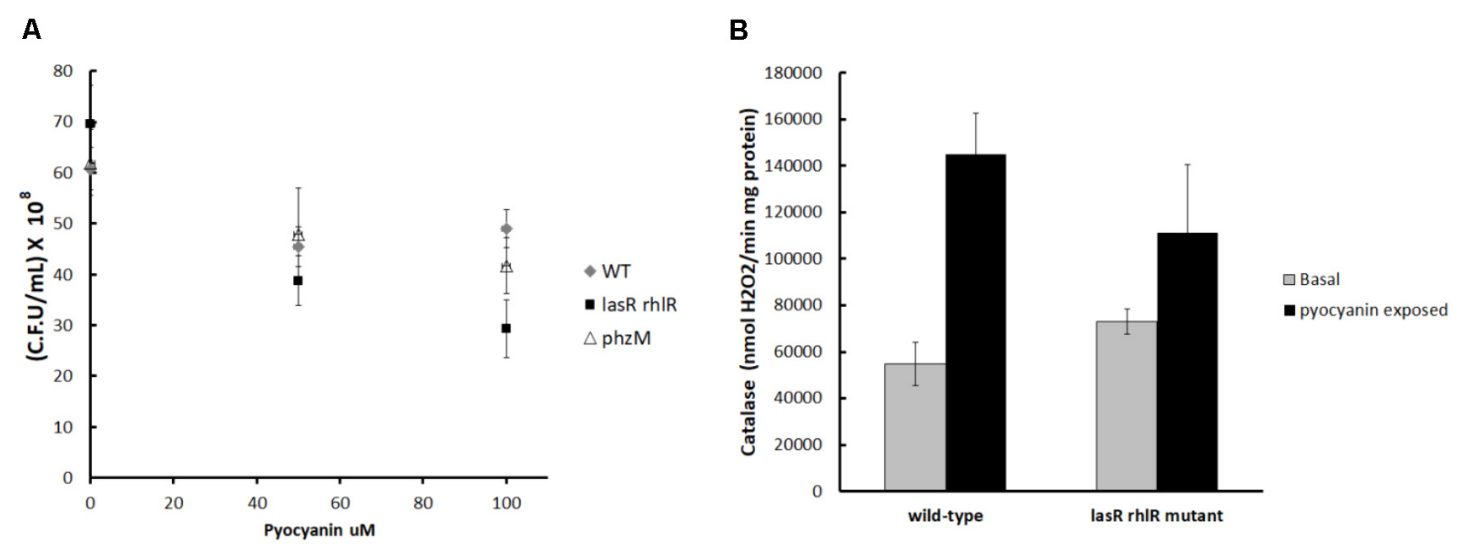

FIGURE 4 | (A) Bactericidal effect of pyocyanin on PA14, the lasR rh/R mutant, and the phzM mutant. Cells were cultured in LB to turbidity at 600 nm of 1.0 , then samples $(1 \mathrm{~mL})$ were taken and pyocyanin was added at the indicated concentrations. Cells were exposed 30 min, and then viable counts were used to determine the degree of survival. Experiments were done in quadruplicate, and the average \pm SEM is shown. The difference between the survival of the wild-type strain and the las $R$ rhIR mutant with $100 \neq \mathrm{M}$ of pyocyanin is significant $(P<0.05$ in a two tailed $T$-student test). (B) Effect of pyocyanin on the catalase activity of PA14 and the las $R$ rhIR mutant. The difference between the catalase activity of the wild-type with and without pyocyanin is significant $(P<0.05$ in a two tailed $T$-student test).

is composed of an initial spatial configuration of agents and allows simulating the interactions of these agents to assess the collective behavior over time and space. This type of model allows to re-create observed behavior but also to predict the appearance of emergent interactions. Our model was composed of the agents, behavioral and interaction rules as well as an environment (a 2D domain in our case) where all takes place. As an ABM allows us to simulate specific scenarios, this results on numerical simulations, the output of such models. In Supplementary Figure SM2, the result of one of such simulations at a fixed time is shown, the output is visualized as a set of microcolonies of wild-type and QS deficient mutants that grow (in two dimensions) by the assimilation of digestible nutrients (peptides and amino acids), derived from nondigestible ones (caseinate) by the action of exoproteases produced only by the wild-type population. In Supplementary Figure SM3, the dynamics of both populations as a function of time in the absence and presence of pyocyanin (which exerts a bactericidal effect only in the QS deficient mutants) are simulated, showing indeed a decrease in the mutant population as an effect of pyocyanin. Furthermore, the model (i) predicts that pyocyanin would have only very subtle effects on the concentrations of autoinducers, caseinate and exoproteases (Supplementary Figure SM4), (ii) shows how pyocyanin concentration changes with time (Supplementary Figure SM5), and (iii) predicts that an increase of the diffusion of the digestible nutrients strongly increases the selection of the QS deficient mutants.

This type of model can help to choose suitable arrangements for future experiments, to explore certain effects visibly and to understand better the behavior of such systems in a more realistic, heterogeneous environment such as biofilms. Evidence suggests that physical properties of the environment may play an important role in competition (Mund et al., 2017).

\section{Pyocyanin Restricts the Appearance of Natural Selected Social Cheaters}

In addition to the short time competition experiments, we decided to test if pyocyanin could restrict the appearance of social cheaters in long term experiments in a population that was entirely composed of wild-type individuals at the beginning. For this, cultures in M9 caseinate medium were started from a single wild-type colony and several consecutive daily subcultures in the same medium were done with or without the addition of $100 \mu \mathrm{M}$ of pyocyanin before each pass. As expected, after eight culture passes, protease-less mutants (cheaters) began to appear and their proportion increased until pass number fourteen; however, in agreement with our previous experiments, the addition of pyocyanin significantly decreased the proportion of social cheaters at days 8,12 , and 14 (Figure 5A). As expected, pyocyanin addition also decreased the cheater proportion in cultures initiated with the phzM mutant (Figure 5B); nevertheless, these differences were not significant and the proportion of cheaters that emerged in the phzM background was lower than the observed for the wild-type strain.

\section{Pyocyanin Selects Active QS in A. baumannii}

After demonstrating that pyocyanin selected for active QS in $P$. aeruginosa, we also tested its effect in another bacterial species, A. baumannii, an important nosocomial pathogen able to coexist with $P$. aeruginosa and a strain that upregulates its catalase activity via QS. It was previously shown that the deletion of abaI impairs production of homoserine lactone and increases sensitivity to pyocyanin (Bhargava et al., 2014). To test if pyocyanin could also select QS in A. baumannii, competitions between the wild-type ATCC17978 strain and its isogenic abaI mutant were conducted by mixing equal quantities of both strains grown in LB (in which they grow with virtually identical growth 

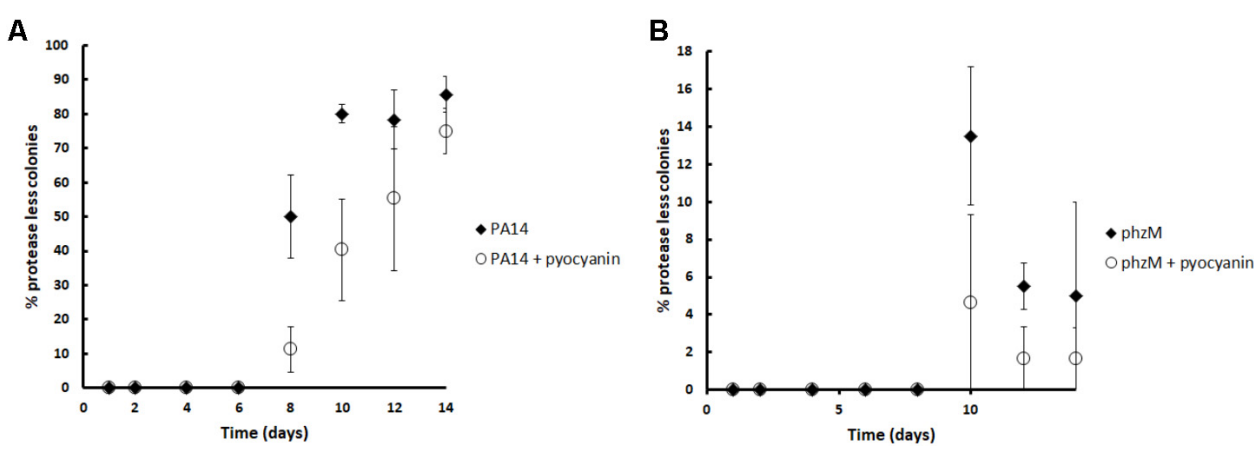

FIGURE 5 | Pyocyanin addition decreases the protease-less individuals (social cheaters) in casein cultures. Experiments were done in triplicate, and the average is shown. Pyocyanin $(100 \mu \mathrm{M})$ was added to the medium before each daily inoculation, and subsequent cultures were done every $24 \mathrm{~h}$. Experiments were done in triplicate, and the average \pm SEM is shown. The difference between the control and the pyocyanin curve was significant at 8 , 10, and 14 days according a Chi-squared test $(P<0.05)$.

rates of $1 \pm 0.05$ and $1.03 \pm 0.01 \mathrm{~h}^{-1}$, Supplementary Figures $\mathrm{S} 2 \mathrm{~A}, \mathrm{C})$ and growing to the exponential phase and by incubating for $20 \mathrm{~min}$ with $50 \mu \mathrm{M}$ of pyocyanin. These competitions were made only at short times to avoid sensing of the wild-typeproduced homoserine lactone by the abaI mutant. In order to estimate the initial and final proportions of the strains, PCR amplification of the abaI gene was performed. Our results showed that without pyocyanin, the initial mutant proportion remained stable, whereas the addition of pyocyanin decreased the mutant to around half the initial proportion (Figure 6). These results, as for $P$. aeruginosa, correlate with higher catalase activity of the wild-type strain relative to the activity of the QS defective mutant and with a significant induction of catalase by the addition of pyocyanin only in the case of the wild-type strain (Supplementary Figure S3). Hence, we found that the pyocyanin produced by $P$. aeruginosa can select for QS in another bacterial system; therefore, the influence of pyocyanin in bacterial ecology may be broad.

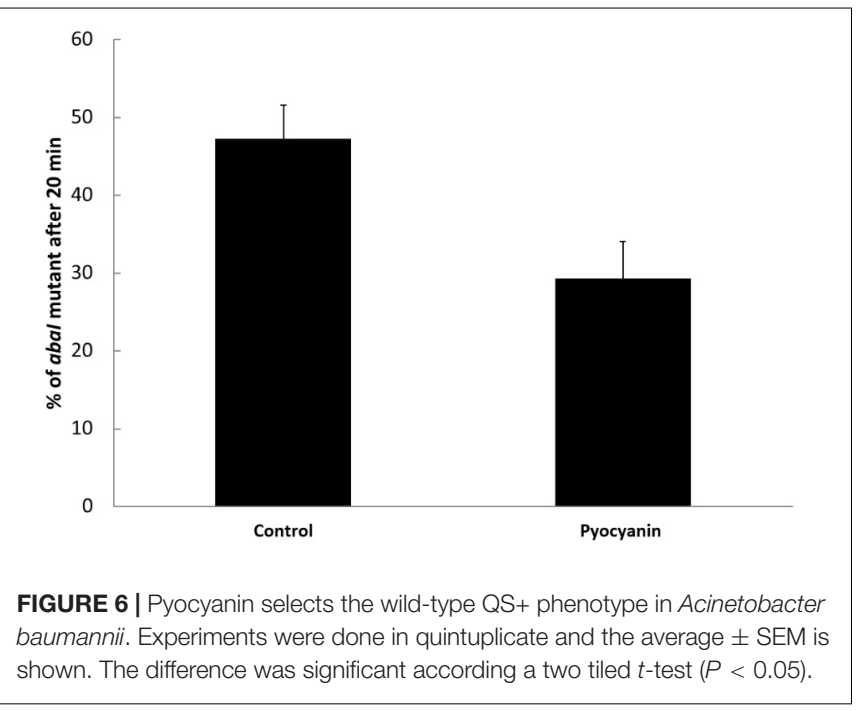

\section{DISCUSSION}

In this work, we demonstrated that pyocyanin is able to select functional QS systems in both $P$. aeruginosa and A. baumannii. The toxicity of pyocyanin is due its generation of oxygen-reactive species, including $\mathrm{H}_{2} \mathrm{O}_{2}$, that cause cell lysis (Das and Manefield, 2012), Our results are in concordance with the fact that in $P$. aeruginosa, QS mutants are more sensitive to several kinds of stress than the strains with functional QS systems (García-Contreras et al., 2015b). These stresses include heat shock, heavy metal stress, and oxidative stress by $\mathrm{H}_{2} \mathrm{O}_{2}$ (García-Contreras et al., 2015b). Hence, the addition of $\mathrm{H}_{2} \mathrm{O}_{2}$ selects for active QS systems during growth on casein as the sole carbon source, which requires the production of QS-controlled exoproteases (García-Contreras et al., 2015b). The lower tolerance of QS mutants to stress may be linked to a deficient expression of antioxidant enzymes during an insult (García-Contreras et al., 2015b), as well to other phenomena such as the production of less robust cell membranes by the QS deficient individuals (Davenport et al., 2015). Critically, stress in general is omnipresent in the variable natural environments of bacteria, and oxidative stress in particular is a common strategy used to fight bacterial pathogens in eukaryotes (Hampton et al., 1998; Abramovitch and Martin, 2004; Vallet-Gely et al., 2008).

Interestingly, a recent publication in which the role of nitrogen source in the selection of social cheaters was evaluated; it shows that in those cultures in which the population remained stable (tragedy of the commons was avoided), the cooperators evolved by decreasing their exoprotease production and by increasing their production of pyocyanin (Lai et al., 2018). This result complements our findings by suggesting that overexpression of this toxin may be a natural strategy to counteract social cheating.

Although for this work we did not explore the effect of pyocyanin in competitions under conditions in which cheating is not present, pyocyanin may also select the wildtype phenotype under these kinds of conditions over QS 
defective mutants. Moreover, other redox active phenazines like phenazine-1-carboxylic acid may also participate in QS selection, but this remains to be explored. In addition, there may be possible contributions to the observed effects mediated by the pyocyanin biosynthetic intermediate 5-methyl phenazine-1carboxylic acid since the phzM mutant also is deficient in its production.

Intriguingly, although we predicted that a higher number of cheaters would arise in the case of $p h z M$ cultures than in the wildtype cultures (since the wild-type strain produces pyocyanin), we observed the opposite (Figure 5B). We speculate that this result may be caused by a lower exoprotease activity of the phzM mutant compare to the parental strain. Accordingly, the phzM mutant has significantly lower collagenase and elastase activities (data not shown) which will decrease cheater fitness. In addition, we cannot rule out the presence of compensatory mechanisms that counteract the cheater fitness, like perhaps a higher synthesis of HCN. Nevertheless, this hypothesis needs to be tested.

Generally, various kinds of stress tend to be positively correlated with cell density due to competition for resources or the accumulation of waste products. This probably explains why an increasing number of private stress responses are shown to be QS regulated (Cornforth and Foster, 2013; García-Contreras et al., 2015b). We thus hypothesize that - as a side effect one of the main stabilizing mechanisms of cooperation via public goods are QS-regulated private stress responses (GarcíaContreras et al., 2015b), although QS controlled stress responses do not need to be private (Friman et al., 2013; Sun et al., 2015).

Stabilization of cooperation by the QS-regulated stress response acts complementarily to stabilization by metabolic prudence (Xavier et al., 2011). The metabolic prudence concept explains that cells produce public goods preferentially when the cost of their production and impact on individual fitness is low, and under such conditions non-producing cheaters have no fitness benefit. In contrast, our study showed that pleiotropic stabilization of cooperation via public goods also works when cooperative production of public goods is associated with fitness costs for the producer.

Overall, we demonstrate that although cheating decreases the fitness of QS proficient individuals in the absence of stress, the higher susceptibility of QS mutants to the pyocyanin produced by the wild-type strain allows for the preservation of functional QS systems; i.e., pyocyanin promotes the selection of more virulent bacteria. Hence, our results provide a new example of social policing by the cooperators and complement the findings of Wang and colleagues that in 2015 showed that QS-controlled production of toxic $\mathrm{HCN}$ by cooperative individuals decreases the fitness of the social cheaters, because social cheaters are more susceptible to $\mathrm{HCN}$ than cooperators, presumably by the lack of expression of an $\mathrm{HCN}$ insensitive cytochrome oxidase that is positively controlled by QS (Wang et al., 2015).

\section{AUTHOR CONTRIBUTIONS}

PC-T, JR-P, MS-M, YG, and MT performed the experiments. JP-V, CK, and AJ made the mathematical model. RG-C, JP-V, CK, TM, JJ-C, and TW conceived the study. RG-C, JP-V, CK, and TW wrote the manuscript. JJ-C analyzed the data.

\section{FUNDING}

This work was supported by grants from PAPIIT UNAM No. IA201116, PAPIIT UNAM IN 214218, and Projects for Solving National Problems/CONACyT Mexico No. 2015-01-402 for RG-C, and by the Army Research Office (W911NF-14-1-0279) to TW, and PI16/01163, awarded to MT within the State Plan for R+D+I 2013-2016 (National Plan for Scientific Research, Technological Development and Innovation 2008-2011) and co-financed by the ISCIIIDeputy General Directorate of evaluation and Promotion of Research - European Regional Development Fund "A way of Making Europe" and Instituto de Salud Carlos III FEDER, Spanish Network for the Research in Infectious Diseases (REIPI, RD16/0016/0001 and RD16/0016/0006) as well as the Study Group on Mechanisms of Action and Resistance to Antimicrobials, GEMARA (SEIMC, http://www.seimc.org/). MT was financially supported by the Miguel Servet Research Program (SERGAS and ISCIII). AJ thanks DAAD (Deutscher Akademischer Austauschdienst), for funding his stay at Technische Universität München, Munich, Germany. TW is the Biotechnology Endowed Chair at the Pennsylvania State University.

\section{ACKNOWLEDGMENTS}

We thank Dr. Frederick Ausubel from the Harvard Medical School and Dr. You-Hee Cho from the College of Pharmacy, CHA University, South Korea for the PA14 strains. We are grateful for the help of Abigail González-Valdez with the transfection of the phzM mutation. RG-C thanks Beatriz MerazRios, Yahira Katherine Porras Hernandez, Berenice PerezEretza, and Dánae Díaz Ramírez for their assistance with some experiments. In addition, we are also grateful for the insights and inspiration provided by our dear colleague Prof. Burkard Hense (who sadly passed away last year). He was very involved at the initial stages of this project; this manuscript honors his memory.

\section{SUPPLEMENTARY MATERIAL}

The Supplementary Material for this article can be found online at: https://www.frontiersin.org/articles/10.3389/fmicb. 2018.01348/full\#supplementary-material 


\section{REFERENCES}

Abramovitch, R. B., and Martin, G. B. (2004). Strategies used by bacterial pathogens to suppress plant defenses. Curr. Opin. Plant Biol. 7, 356-364. doi: 10.1016/j.pbi. 2004.05.002

Bhargava, N., Sharma, P., and Capalash, N. (2014). Pyocyanin stimulates quorum sensing-mediated tolerance to oxidative stress and increases persister cell populations in Acinetobacter baumannii. Infect. Immun. 82, 3417-3425. doi: 10.1128/IAI.01600-14

Budzik, J. M., Rosche, W. A., Rietsch, A., and O’Toole, G. A. (2004). Isolation and characterization of a generalized transducing phage for Pseudomonas aeruginosa strains PAO1 and PA14. J. Bacteriol. 186, 3270-3273. doi: 10.1128/ JB.186.10.3270-3273.2004

Caldwell, C. C., Chen, Y., Goetzmann, H. S., Hao, Y., Borchers, M. T., Hassett, D. J., et al. (2009). Pseudomonas aeruginosa exotoxin pyocyanin causes cystic fibrosis airway pathogenesis. Am. J. Pathol. 175, 2473-2488. doi: 10.2353/ajpath.2009. 090166

Castillo-Juarez, I., Maeda, T., Mandujano-Tinoco, E. A., Tomas, M., PerezEretza, B., Garcia-Contreras, S. J., et al. (2015). Role of quorum sensing in bacterial infections. World J. Clin. Cases 3, 575-598. doi: 10.12998/wjcc.v3. i7. 575

Cornforth, D. M., and Foster, K. R. (2013). Competition sensing: the social side of bacterial stress responses. Nat. Rev. Microbiol. 11, 285-293. doi: 10.1038/ nrmicro2977

Dandekar, A. A., Chugani, S., and Greenberg, E. P. (2012). Bacterial quorum sensing and metabolic incentives to cooperate. Science 338, 264-266. doi: 10.1126/science.1227289

Dandekar, A. A., Chugani, S., and Greenberg, E. P. (2013). Bacterial quorum sensing and metabolic incentives to cooperate. Science 338, 264-266. doi: 10. 1126/science. 1227289

Das, T., and Manefield, M. (2012). Pyocyanin promotes extracellular DNA release in Pseudomonas aeruginosa. PLoS One 7:e46718. doi: 10.1371/journal.pone. 0046718

Davenport, P. W., Griffin, J. L., and Welch, M. (2015). Quorum sensing is accompanied by global metabolic changes in the opportunistic human pathogen Pseudomonas aeruginosa. J. Bacteriol. 197, 2072-2082. doi: 10.1128/JB.02 557-14

Dekimpe, V., and Deziel, E. (2009). Revisiting the quorum-sensing hierarchy in Pseudomonas aeruginosa: the transcriptional regulator RhlR regulates LasRspecific factors. Microbiology 155, 712-723. doi: 10.1099/mic.0.022764-0

Dietrich, L. E., Price-Whelan, A., Petersen, A., Whiteley, M., and Newman, D. K. (2006). The phenazine pyocyanin is a terminal signalling factor in the quorum sensing network of Pseudomonas aeruginosa. Mol. Microbiol. 61, 1308-1321. doi: 10.1111/j.1365-2958.2006.05306.x

Diggle, S. P., Griffin, A. S., Campbell, G. S., and West, S. A. (2007). Cooperation and conflict in quorum-sensing bacterial populations. Nature 450, 411-414. doi: $10.1038 /$ nature06279

Dumas, Z., and Kummerli, R. (2012). Cost of cooperation rules selection for cheats in bacterial metapopulations. J. Evol. Biol. 25, 473-484. doi: 10.1111/j.14209101.2011.02437.x

Essar, D. W., Eberly, L., Hadero, A., and Crawford, I. P. (1990). Identification and characterization of genes for a second anthranilate synthase in Pseudomonas aeruginosa: interchangeability of the two anthranilate synthases and evolutionary implications. J. Bacteriol. 172, 884-900. doi: 10.1128/jb.172.2. 884-900.1990

Friman, V. P., Diggle, S. P., and Buckling, A. (2013). Protist predation can favour cooperation within bacterial species. Biol. Lett. 9:20130548. doi: 10.1098/rsbl. 2013.0548

García-Contreras, R. (2016). Is quorum sensing interference a viable alternative to treat Pseudomonas aeruginosa infections? Front. Microbiol. 7:1454. doi: 10. 3389/fmicb.2016.01454

García-Contreras, R., Lira-Silva, E., Jasso-Chavez, R., Hernandez-Gonzalez, I. L., Maeda, T., Hashimoto, T., et al. (2013). Isolation and characterization of gallium resistant Pseudomonas aeruginosa mutants. Int. J. Med. Microbiol. 303, 574-582. doi: 10.1016/j.ijmm.2013.07.009

García-Contreras, R., Nunez-Lopez, L., Jasso-Chavez, R., Kwan, B. W., Belmont, J. A., Rangel-Vega, A., et al. (2015a). Quorum sensing enhancement of the stress response promotes resistance to quorum quenching and prevents social cheating. ISME J. 9, 115-125. doi: 10.1038/ismej.2014.98

García-Contreras, R., Perez-Eretza, B., Jasso-Chavez, R., Lira-Silva, E., RoldanSanchez, J. A., Gonzalez-Valdez, A., et al. (2015b). High variability in quorum quenching and growth inhibition by furanone C-30 in Pseudomonas aeruginosa clinical isolates from cystic fibrosis patients. Pathog. Dis. 73:ftv040. doi: 10.1093/ femspd/ftv040

Gerdt, J. P., and Blackwell, H. E. (2014). Competition studies confirm two major barriers that can preclude the spread of resistance to quorum-sensing inhibitors in bacteria. ACS Chem. Biol. 9, 2291-2299. doi: 10.1021/cb5004288

Grosso-Becerra, M. V., Santos-Medellin, C., Gonzalez-Valdez, A., Mendez, J. L., Delgado, G., Morales-Espinosa, R., et al. (2014). Pseudomonas aeruginosa clinical and environmental isolates constitute a single population with high phenotypic diversity. BMC Genomics 15:318. doi: 10.1186/1471-216415-318

Guendouze, A., Plener, L., Bzdrenga, J., Jacquet, P., Remy, B., Elias, M., et al. (2017). Effect of quorum quenching lactonase in clinical isolates of Pseudomonas aeruginosa and comparison with quorum sensing inhibitors. Front. Microbiol. 8:227. doi: 10.3389/fmicb.2017.00227

Hamad, M. A., Zajdowicz, S. L., Holmes, R. K., and Voskuil, M. I. (2009). An allelic exchange system for compliant genetic manipulation of the select agents Burkholderia pseudomallei and Burkholderia mallei. Gene 430, 123-131. doi: 10.1016/j.gene.2008.10.011

Hampton, M. B., Kettle, A. J., and Winterbourn, C. C. (1998). Inside the neutrophil phagosome: oxidants, myeloperoxidase, and bacterial killing. Blood 92, 30073017.

Hassett, D. J., Ma, J. F., Elkins, J. G., McDermott, T. R., Ochsner, U. A., West, S. E., et al. (1999). Quorum sensing in Pseudomonas aeruginosa controls expression of catalase and superoxide dismutase genes and mediates biofilm susceptibility to hydrogen peroxide. Mol. Microbiol. 34, 1082-1093. doi: 10.1046/j.1365-2958. 1999.01672.x

Hense, B. A., Kuttler, C., Muller, J., Rothballer, M., Hartmann, A., and Kreft, J. U. (2007). Does efficiency sensing unify diffusion and quorum sensing? Nat. Rev. Microbiol. 5, 230-239.

Hoffman, L. R., Kulasekara, H. D., Emerson, J., Houston, L. S., Burns, J. L., Ramsey, B. W., et al. (2009). Pseudomonas aeruginosa lasR mutants are associated with cystic fibrosis lung disease progression. J. Cyst. Fibros. 8, 66-70. doi: 10.1016/j. jcf.2008.09.006

Howe, T. R., and Iglewski, B. H. (1984). Isolation and characterization of alkaline protease-deficient mutants of Pseudomonas aeruginosa in vitro and in a mouse eye model. Infect. Immun. 43, 1058-1063.

Kummerli, R., Griffin, A. S., West, S. A., Buckling, A., and Harrison, F. (2009). Viscous medium promotes cooperation in the pathogenic bacterium Pseudomonas aeruginosa. Proc. R. Soc. B Biol. Sci. 276, 3531-3538. doi: 10.1098/ rspb.2009.0861

Lai, B. M., Yan, H. C., Wang, M. Z., Li, N., and Shen, D. S. (2018). A common evolutionary pathway for maintaining quorum sensing in Pseudomonas aeruginosa. J. Microbiol. 56, 83-89. doi: 10.1007/s12275-018-7286-1

Lee, J., and Zhang, L. (2015). The hierarchy quorum sensing network in Pseudomonas aeruginosa. Protein Cell 6, 26-41. doi: 10.1007/s13238-0140100-x

Liberati, N. T., Urbach, J. M., Miyata, S., Lee, D. G., Drenkard, E., Wu, G., et al. (2006). An ordered, nonredundant library of Pseudomonas aeruginosa strain PA14 transposon insertion mutants. Proc. Natl. Acad. Sci. U.S.A. 103, 2833-2838. doi: 10.1073/pnas.0511100103

Mellbye, B., and Schuster, M. (2011). The sociomicrobiology of antivirulence drug resistance: a proof of concept. mBio 2:e00131-11. doi: 10.1128/mBio. 00131-11

Muller, M., and Merrett, N. D. (2014). Pyocyanin production by Pseudomonas aeruginosa confers resistance to ionic silver. Antimicrob. Agents Chemother. 58, 5492-5499. doi: 10.1128/AAC.03069- 14

Mund, A., Diggle, S. P., and Harrison, F. (2017). The fitness of Pseudomonas aeruginosa quorum sensing signal cheats is influenced by the diffusivity of the environment. mBio 8:e00353-17. doi: 10.1128/mBio.00353-17

Mund, A., Kuttler, C., Perez-Velazquez, J., and Hense, B. A. (2016). An agedependent model to analyse the evolutionary stability of bacterial quorum sensing. J. Theor. Biol. 405, 104-115. doi: 10.1016/j.jtbi.2015.12.021 
Ohman, D. E., Cryz, S. J., and Iglewski, B. H. (1980). Isolation and characterization of Pseudomonas aeruginosa PAO mutant that produces altered elastase. J. Bacteriol. 142, 836-842.

O’Malley, Y. Q., Reszka, K. J., Spitz, D. R., Denning, G. M., and Britigan, B. E. (2004). Pseudomonas aeruginosa pyocyanin directly oxidizes glutathione and decreases its levels in airway epithelial cells. Am. J. Physiol. Lung Cell. Mol. Physiol. 287, L94-L103. doi: 10.1152/ajplung.00025.2004

Papenfort, K., and Bassler, B. L. (2016). Quorum sensing signal-response systems in Gram-negative bacteria. Nat. Rev. Microbiol. 14, 576-588. doi: 10.1038/nrmicro. 2016.89

Park, S. Y., Heo, Y. J., Choi, Y. S., Deziel, E., and Cho, Y. H. (2005). Conserved virulence factors of Pseudomonas aeruginosa are required for killing Bacillus subtilis. J. Microbiol. 43, 443-450.

Popat, R., Cornforth, D. M., McNally, L., and Brown, S. P. (2015). Collective sensing and collective responses in quorum-sensing bacteria. J. R. Soc. Interface 12:20140882. doi: 10.1098/rsif.2014.0882

Rada, B., and Leto, T. L. (2013). Pyocyanin effects on respiratory epithelium: relevance in Pseudomonas aeruginosa airway infections. Trends Microbiol. 21, 73-81. doi: 10.1016/j.tim.2012.10.004

Ramos, I., Dietrich, L. E., Price-Whelan, A., and Newman, D. K. (2010). Phenazines affect biofilm formation by Pseudomonas aeruginosa in similar ways at various scales. Res. Microbiol. 161, 187-191. doi: 10.1016/j.resmic.2010. 01.003

Rezzoagli, C., Wilson, D., Weigert, M., Wyder, S., and Kummerli, R. (2017). Probing the evolutionary robustness of anti-virulence treatments targeting iron uptake in Pseudomonas aeruginosa. bioRxiv [Preprint]. doi: 10.1101/195974

Sandoz, K. M., Mitzimberg, S. M., and Schuster, M. (2007). Social cheating in Pseudomonas aeruginosa quorum sensing. Proc. Natl. Acad. Sci. U.S.A. 104, 15876-15881. doi: 10.1073/pnas.0705653104

Saucedo-Mora, M. A., Castaneda-Tamez, P., Cazares, A., Perez-Velazquez, J., Hense, B. A., Cazares, D., et al. (2017). Selection of functional quorum sensing systems by lysogenic bacteriophages in Pseudomonas aeruginosa. Front. Microbiol. 8:1669. doi: 10.3389/fmicb.2017.01669

Schaber, J. A., Carty, N. L., McDonald, N. A., Graham, E. D., Cheluvappa, R., Griswold, J. A., et al. (2004). Analysis of quorum sensing-deficient clinical isolates of Pseudomonas aeruginosa. J. Med. Microbiol. 53, 841-853. doi: 10. 1099/jmm.0.45617-0
Smalley, N. E., An, D., Parsek, M. R., Chandler, J. R., and Dandekar, A. A. (2015). Quorum sensing protects Pseudomonas aeruginosa against cheating by other species in a laboratory coculture model. J. Bacteriol. 197, 3154-3159. doi: 10. 1128/JB.00482-15

Sun, S., Tay, Q. X., Kjelleberg, S., Rice, S. A., and McDougald, D. (2015). Quorum sensing-regulated chitin metabolism provides grazing resistance to Vibrio cholerae biofilms. ISME J. 9, 1812-1820. doi: 10.1038/ismej.2014.265

Vallet-Gely, I., Lemaitre, B., and Boccard, F. (2008). Bacterial strategies to overcome insect defences. Nat. Rev. Microbiol. 6, 302-313. doi: 10.1038/nrmicro 1870

Wang, M., Schaefer, A. L., Dandekar, A. A., and Greenberg, E. P. (2015). Quorum sensing and policing of Pseudomonas aeruginosa social cheaters. Proc. Natl. Acad. Sci. U.S.A. 112, 2187-2191. doi: 10.1073/pnas.1500704112

Wang, Y., Kern, S. E., and Newman, D. K. (2010). Endogenous phenazine antibiotics promote anaerobic survival of Pseudomonas aeruginosa via extracellular electron transfer. J. Bacteriol. 192, 365-369. doi: 10.1128/JB.01 188-09

Wang, Y., Wilks, J. C., Danhorn, T., Ramos, I., Croal, L., and Newman, D. K. (2011). Phenazine-1-carboxylic acid promotes bacterial biofilm development via ferrous iron acquisition. J. Bacteriol. 193, 3606-3617. doi: 10.1128/JB.00 396-11

Xavier, J. B., Kim, W., and Foster, K. R. (2011). A molecular mechanism that stabilizes cooperative secretions in Pseudomonas aeruginosa. Mol. Microbiol. 79, 166-179. doi: 10.1111/j.1365-2958.2010.07436.x

Conflict of Interest Statement: The authors declare that the research was conducted in the absence of any commercial or financial relationships that could be construed as a potential conflict of interest.

Copyright (c) 2018 Castañeda-Tamez, Ramírez-Peris, Pérez-Velázquez, Kuttler, Jalalimanesh, Saucedo-Mora, Jiménez-Cortés, Maeda, González, Tomás, Wood and García-Contreras. This is an open-access article distributed under the terms of the Creative Commons Attribution License (CC BY). The use, distribution or reproduction in other forums is permitted, provided the original author(s) and the copyright owner are credited and that the original publication in this journal is cited, in accordance with accepted academic practice. No use, distribution or reproduction is permitted which does not comply with these terms. 